\title{
Immersive Virtual Training Environment for Teaching Single- and Multi- queuing Theory: Industrial Engineering Queuing Theory Concepts
}

\author{
Dr. Michael Andre Hamilton, Mississippi State University
}

Dr. Michael A. Hamilton is the Associate Director at Mississippi State Institute for System Engineering Research (ISER) in Vicksburg, MS. He received his Doctorate, Master's and Bachelor's degrees in Industrial and Systems Engineering from Mississippi State University and has a graduate certificate in Modeling, Simulation, and Visualization Engineering from Old Dominion University. He also received two certifications in Big Data Analytics from University of California, San Diego and Data Science from John Hopkins University. He worked several years in the printing manufacturing industry where he served in numerous positions such as a Manufacturing Engineer, Global Expansion Engineer, and the Manager of Production Operations for the Memphis Division at Mimeo.com.

While attending Mississippi State, Dr. Hamilton developed in-depth research in the effects of obesity in assembly manufacturing and minimizing disruption of freight and passenger flow in the event of terrorist attacks or natural disasters. He has also participated in sponsored research by the Office of Naval Research (ONR) investigating the effects of ship motion on human performance aboard the Littoral Combat Ship, developing human performance algorithms to minimize the effects of motion, fatigue, and temperate on board ship platform, evaluating the Ammunition Canister Protection for the MRAP MATV, and the ETOWL "Lightening the Warfighter Load" for the Marines Expeditionary Warfighter Squad.

Currently, he's the technical lead for Big Data Analytic and Visualization, and Surrogate Modeling efforts in conjunction with the U.S. Army Engineer Research and Development Center (ERDC).

\section{Dr. Raed Jaradat, Mississippi State University}

Raed M. Jaradat is an Assistant Professor at Mississippi State University. His research interests include engineering management, systems engineering, Risk Management, Reliability for System of Systems, and Systems Simulation. He is a topic leader in systems thinking, including systems theory and complex systems, for the Society for Engineering and Management Systems (SEMS). Prior to joining the doctoral program at ODU, he worked as a systems analyst and operations officer for five years. He is a past proceedings chair of the American Society for Engineering Management and is currently a member of the Academy of Management and Institute of Industrial Engineers. He holds a BS in Business, an MA in Operations Management, and $\mathrm{PhD}$ in Engineering Management from Old Dominion University.

Mr. Parker Jones, Mississippi State University

BS Industrial Engineering

\section{Emily S. Wall , Mississippi State University}

Emily Wall is a summa cum laude Industrial and Systems Engineering 2015 graduate of Mississippi State University, and a 2012 graduate of Itawamba Community College. After college, she accepted a position as a Research Engineer at Mississippi State University's Institute for Systems Engineering Research, located in Vicksburg, MS, on the campus of the Engineering Research and Development Center. While at ISER, her research areas include lean six sigma practices and applications, manufacturability, virtual reality applications and Mississippi economic improvement projects. She is a certified Six Sigma Black Belt and is a graduate student at Mississippi State University pursuing her doctorate in Industrial Engineering.

Mr. Vidanelage Lakshika Dayarathna, Mississippi State University

Dr. Debisree Ray, Mississippi State University

Ms. Ginnie Shih En Hsu 


\title{
IMMERSIVE VIRTUAL TRAINING ENVIROMENT FOR TEACHING SINGLE AND MULTI-QUEUING THEORY INDUSTRIAL ENGINEERING QUEUING THEORY CONCEPTS
}

\begin{abstract}
:
In the domain of Industrial Engineering (IE), there are several theoretical concepts such as, inventory theory and queuing theory. The implementation of VR technology in the IE domain can benefit students by providing an immersive and interactive environment and presenting a more practical and visual context to the theoretical concepts than can be provided through traditional teaching methods. The proposed VR teaching modules allow students 1) to experience the sense of being present in a virtual queuing system environment that is representative of a real-world engineering situation, 2) to expand their natural perceptive abilities and authentic experience, and 3) to eliminate the need to deal with any expensive equipment or risky environments to understand the application of queuing theory. The purpose of the research is to investigate how well students gained conceptual knowledge of queuing theory using the developed VR teaching module. Queueing theory is defined as the mathematical study of waiting lines. In this study students were provided a conceptual queuing theory quiz after the VR teaching module, and then they performed the NASA-TLX to evaluate their perceived workload and effort in competing conceptual quiz. Results showed that students performed well on the theoretical conceptual quiz, and the overall perceived work effort was mentally and physically low.
\end{abstract}

\section{Introduction}

An extensive review of the education literature showed that studies related to VR application found to be in the following areas: effectiveness of VR in education, comparisons of different VR tools used in education, the role of teachers in VR-based learning environments, results in changing the primary role from teacher to facilitator, how children interact with 3D environments, and characteristics that are necessary for spatial learning environments [1]. The literature is replete with reasons for using VR in learning and specifically how it gives the user a sense of "being there" [2]. VR also allows students to have 'hands-on experiences' with real-world engineering situations, and provides the student with a first-person approach, this allows natural affordances for interacting with the environment in a realistic manner.

VR application has spanned the healthcare, aerospace, physics and other domain areas. For example, a pilot study, to evaluate the usefulness of VR in education, was performed using fiftynine students, in a summer day camp. Results indicated that VR is beneficial to students in a learning environment [3]. In another study, fifty-one physics students completed a quasiexperimental study to compare the effectiveness of virtual environments against the teaching methods that are currently used to teach radioactivity [4]. Results showed that the virtual environment was more effective and the students retained and understood the information presented better than the current teaching methods. A ten-year review of empirical research on educational virtual environments was done in 2011 to review the published work in VR up to this 
point. They found that the features (3D spatial representations, multisensory channels for user interaction, immersion and intuitive interaction through natural manipulation in real time) contribute to positive learning outcomes when combined together with other factors of the learning process [5]. A quasi-experimental study to evaluate the effectiveness of VR simulation as a teaching strategy for better nursing skills was performed with twenty students in a first level "Fundamentals of Nursing" course; and a similar experiment comparing traditional instruction vs. virtual reality simulation using nursing students. [6], [7], [8]. Other studies found to be focused more on the role of the teacher in VR learning environments [9].

VR could also be used for breaching threshold concepts that block students from grasping new material [10]. The threshold concept has three dimensions: routine (i.e. boring), irrelevant material, and concept difficulty. After passing through a threshold concept, students have an easier time learning related material with a great desire. VR could help in breaching a threshold concept by presenting information through a new medium. Experiencing how a concept works in a simulation that is familiar to their own lives could also convey how the material is relevant. According to Ausubel's Assimilation Learning Theory, "the most important single factor influencing learning is what the learner already knows" and over time a new concept can be learned by understanding how it relates to previously acquired knowledge [11]. Virtual reality may be able to draw out the user's pre-existing knowledge about their everyday physical world and better integrate new information about relevant engineering concepts

The aim of this study was to investigate how well undergraduate and graduate students gained conceptual knowledge of queuing theory using the developed VR teaching module. Queueing theory is defined as the mathematical study of waiting lines. The notation for a single node described using Kendall's notation A/S/C in which A- represents the distribution of time between arrivals, S- the distribution of service time, and C- the number of servers available [12]. The research objective is presented below, followed by the research approach and the immersive experience of the virtual teaching module.

\section{Research Objective}

The primary objective of this study was to quantify learning effect of student conceptual knowledge through teaching single and multi-queuing theory using an immersive virtual teaching environment. Specifically, this study quantified the learning effects with a conceptual knowledge quiz after conducting the virtual teaching module. The simulation was designed to teach the queuing theory concept to students with little to knowledge about the topic. The quiz is divided into two parts which consisted of 15 conceptual questions and 5 analytical questions (See Appendix A). The secondary objective was to understand students' perceived workload to complete the conceptual knowledge quiz using the NASA TLX. 


\section{Previous studies (Phase I), current studies (Phase II), and future studies (Phase III)}

In a previous study (phase I) we tested whether the VR teaching module would improve students' knowledge gain and motivation compared to traditional teaching methods specific to the queuing theory concept. Thirty-six undergraduate and graduate students from Mississippi State University participated in the research. Pre- and post-knowledge exercises were designed to test the gain in understanding of queueing theory concepts and to test the motivation level using the VR environment. Using multivariate paired Hotelling's T-square test at 0.05 significant level, paired t-test, the non-parametric tests - Kolmogorov Smirnov (KS) and the Mann-Whitney test, the results showed that the proposed VR teaching module outperforms the traditional teaching methods in terms of students' motivation and knowledge gain. The Motivated Strategies for Learning Questionnaire (MSLQ) was used to collect data related to motivation. The results were promising and thus, in this study (phase II) we improved and updated the design of the VR teaching module to be more immersive and visualized. The current VR module is a semi self-paced tutorial.

Concurrent research (Phase III) is being conducted to investigate how well students understand the queuing theory concept using this updated VR teaching module versus traditional classroom lecture. Data is currently being collected using a different set of students with the same conceptual quiz but taught the topic in a traditional classroom manner (control group). Afterwards, we plan to provide a comparative analysis of both approaches, control group versus experimental group and disseminate the results.. The sections discussed below only reflects how well the students perform using the VR training module (experimental group).

\section{Immersive Virtual Teaching Module}

The objective of the learning module was to provide students a learning tool that would teach them simple queuing theory with little to no knowledge of the concept. The VR teaching module was developed using the queuing theory concept and converted the theoretical concept into an immersive virtual experience. The VR headset used for this experiment was the Oculus Rift. The VR teaching module was developed using the Unity3D engine simulation software.

The VR teaching module was developed to teach engineering students single and multi-queueing theory concept. For this purpose, a simulation was developed to represent a food service restaurant. When the student puts on the headset, they are instructed on how to calibrate the eye spacing of the headset lens to optimize the visual image quality. Afterwards, the students are given the Oculus touch controllers and asked to press the "Reach Here to Begin" button to start the simulation. Audio instructions were provided to instruct students on how to move around and use the controllers in the simulation. The left thumb stick moves the student's position in relation to their line of sight and the right thumb stick rotates the student's position. When the student uses both thumb sticks, another button is activated. This button blinks and emits a recurring beep to draw the user's attention. After pressing the button, the queuing theory lecture begins. Figure 1 provides a visual representation of the initial (1-4) steps. 


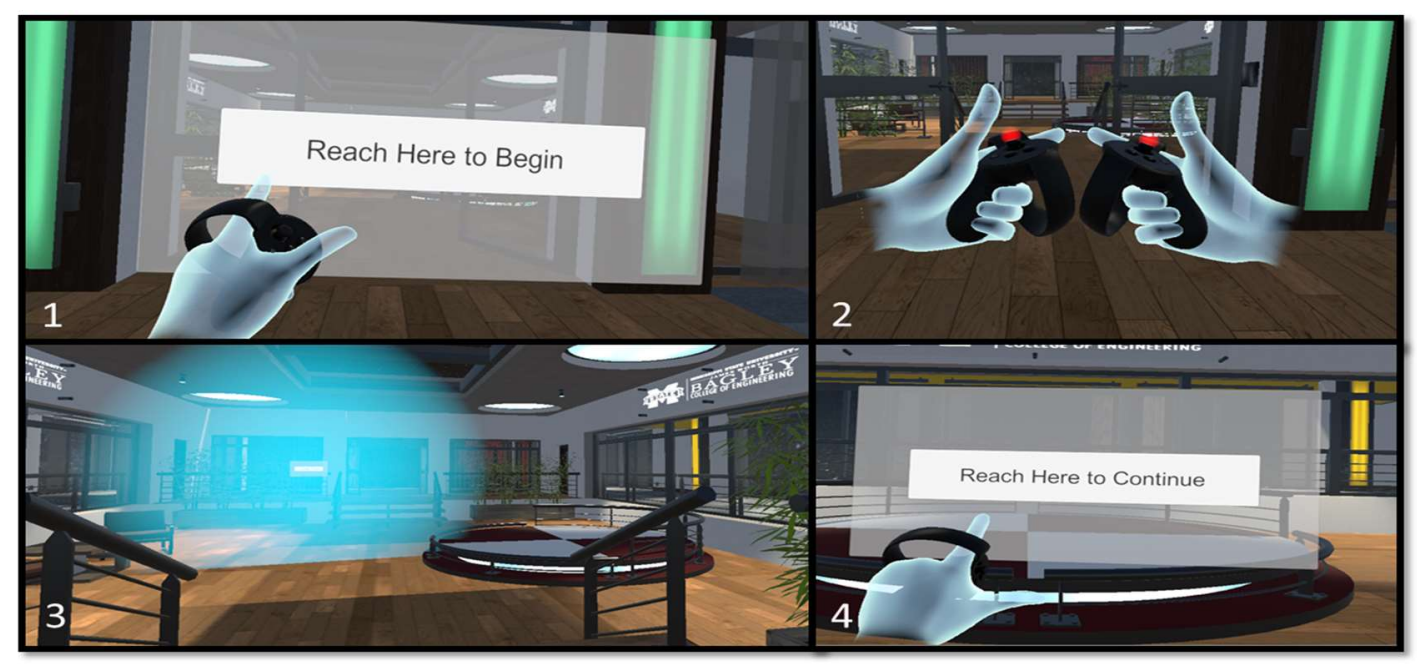

Figure 1: Initialization Steps 1-4

The VR lecture starts with explaining the difference between discrete and continuous data sets. Visual elements are presented to students during the lecture to enhance the understandability of the material. For example, when describing the characteristics of discrete data, a die falls from the ceiling and the text floats to its location to draw attention to the visual element. Likewise, a thermostat is provided during the discussion of continuous data. Figure 2 shows the visual elements that are used for the lecture topic.

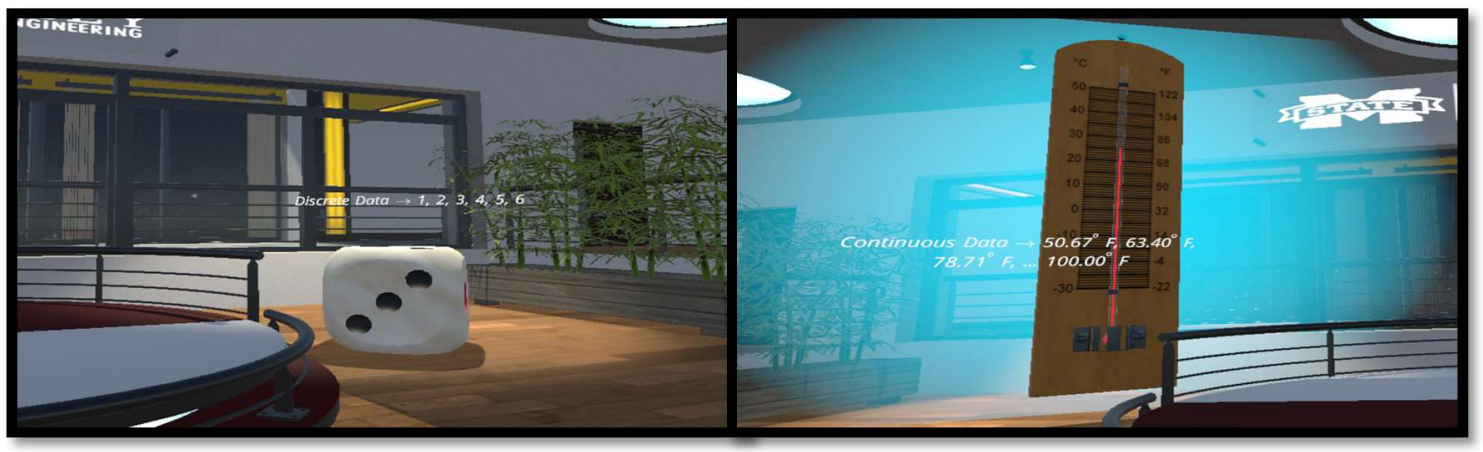

Figure 2: Visual Elements

The next lecture topic is the explanation of the three types of models shown in Figure 3. Similar to the discrete and continuous data discussion, visual elements are used to support the audio portion of the topic. A face (static model), building (physical model), and a sphere represented the portal to teleport you to the mathematical model of queuing theory. When the students interact with the 
sphere, they are transported to the next scene where the remainder of the queuing theory lecture is given.

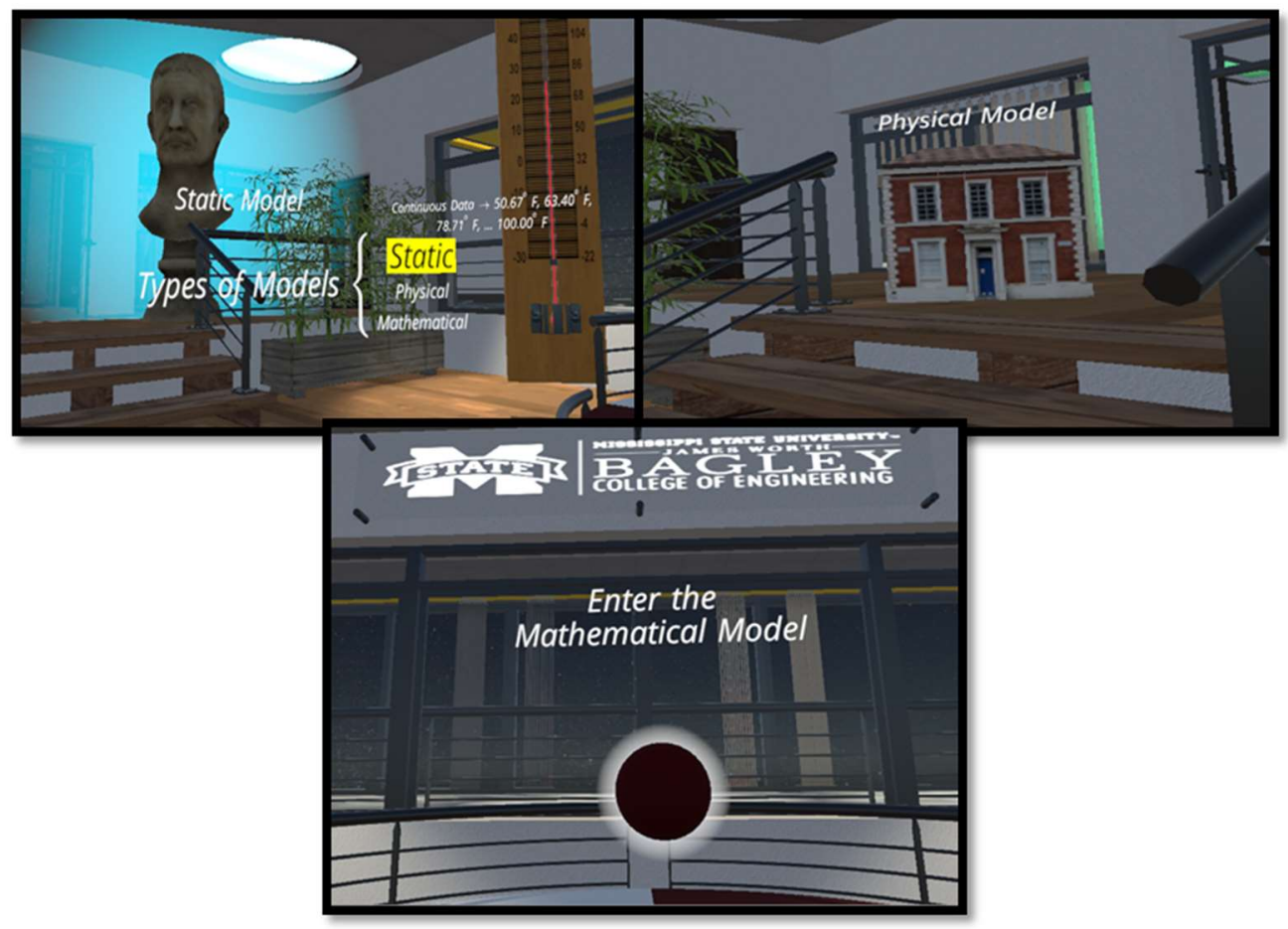

Figure 3: Three Types of Models

After entering the mathematical model portal, the students are integrated into a simulation of a fast-food restaurant. During this portion of the simulation, the theoretical concepts of queuing theory are explained and visual cueing elements are provided to help with the understanding of some theoretical topics. After the lecture portion, students are able to manipulate the simulation by changing the inter-arrival time, number of servers, arrival and service distributions, number of customers entering the simulation, and other parameters. The students watch the effects of the queues in real-time and observed numerous graph options to better understand the effect of the simulation input decision has on the outcome on the queues. Figure 4 shows some of the visual elements that are provided to the students during the duration of the module. 


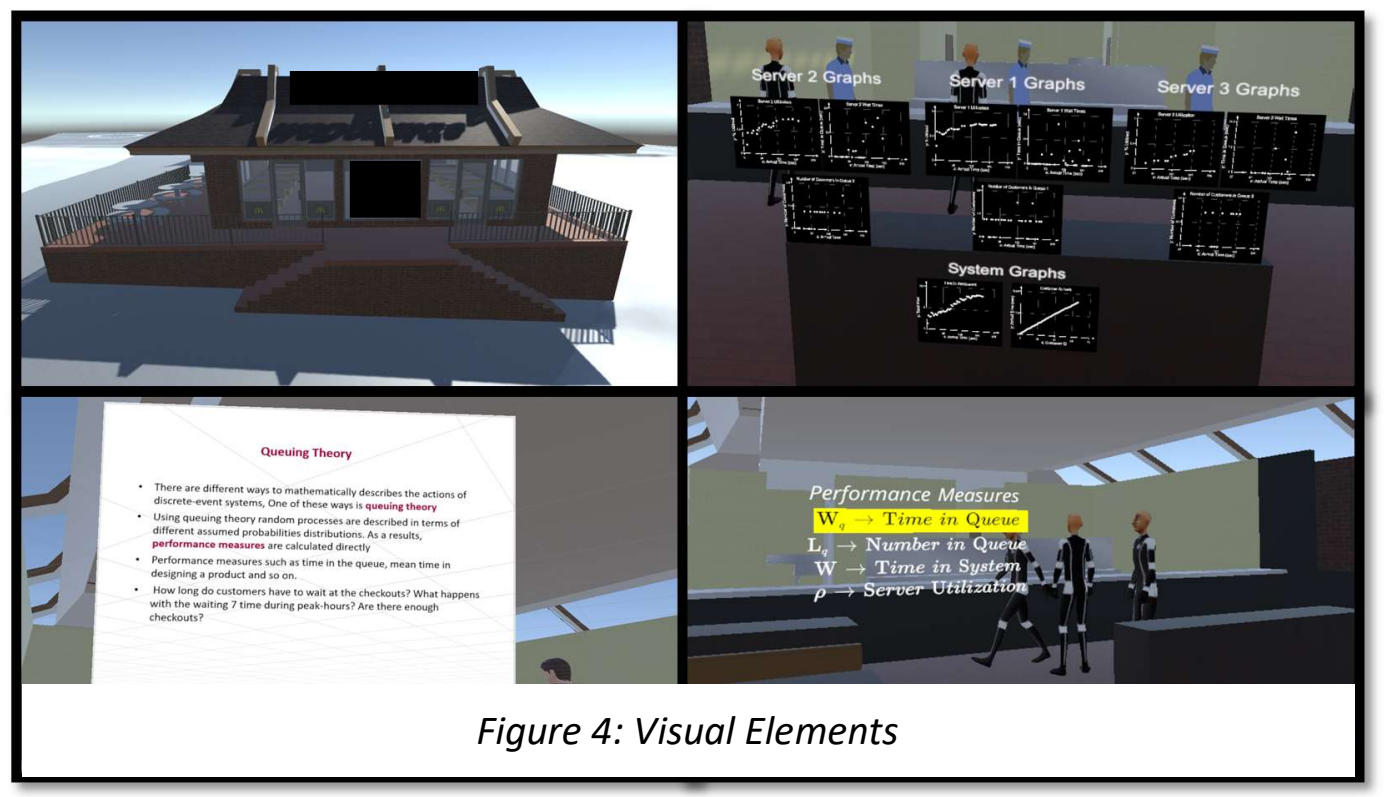

After the lecture is completed, the students complete the quiz within the simulation. They are provided a calculator and the ability to review any of the modules shown throughout the lecture. They also are given hints during the quiz that direct the students to the module that contains the knowledge to answer the question. Figure 5, below, shows the quiz module provided to the students.

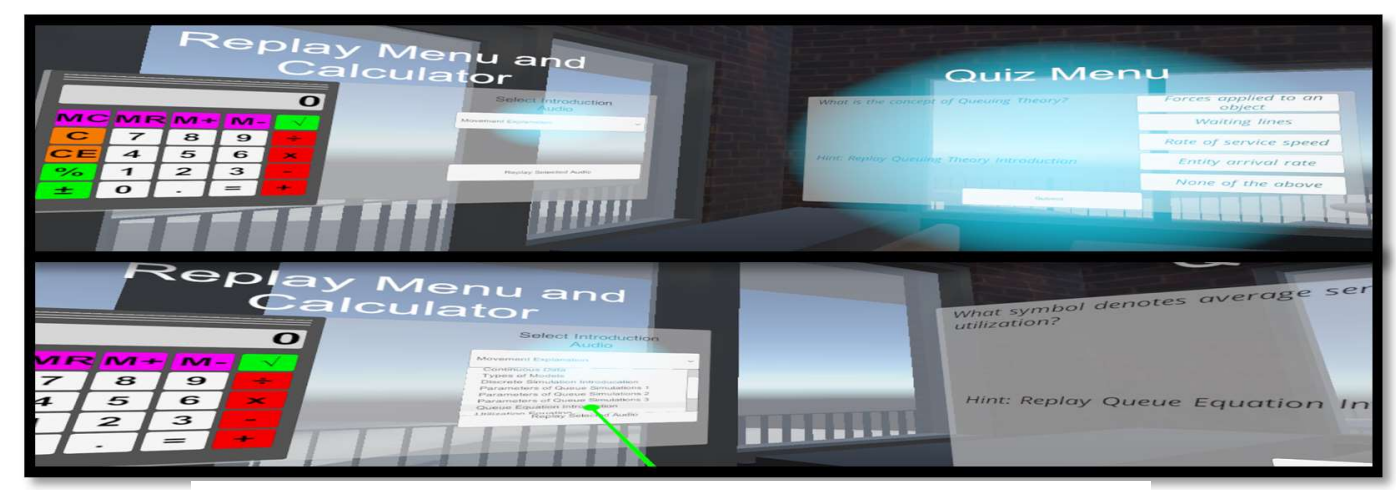

Figure 5: Quiz Module

\section{Method}

The next sub-sections are subdivided based on the demographics, independent variables, the dependent variable, and the NASA TLX assessments. This experiment was collected in a control laboratory environment in which three VR module setups were provided which allowed 3 students to conduct the training at a time. Before starting the simulation, the students were asked to fill out a demographic form and a Likert scale survey to gauge their experience with VR, video games, and previous simulation knowledge. Afterwards, they began using the training module and started 
the simulation tutorial and experiment. For each student, the learning module took appropriately 30 minutes to complete.

\section{Demographics and Independent Variables}

Fifty-six students participated in the study. Ten independent variables were collected. The demographical independent variables were Academic Classification, College Major, Gender, Race, and Origin. None of the independent variables were controlled for this study. All participants had an equal opportunity of involvement in the study. They were selected based on order of arrival. Figure 6, below, shows the distribution of students across the demographic categories.
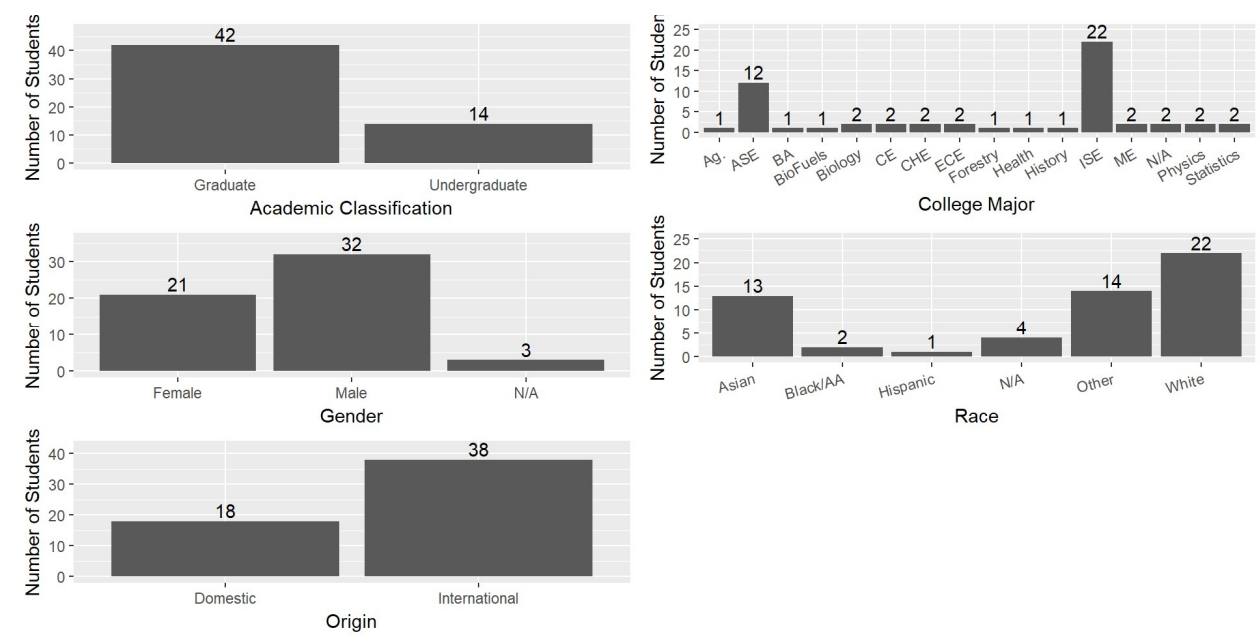

Figure 6: Distribution of Students Across Demographic Categories

$75 \%$ of the participants were graduate students and $39 \%$ of the participants were majoring in Industrial and System Engineering (ISE). Many of the graduate students were unfamiliar with queuing theory concept because their bachelors and master's degrees were in other fields. The international students made up $67 \%$ of the participants and there were slightly more males than females that participated in the effort. The next five independent variables were based on survey questions of the following topics: Statistical Background, System Simulation Knowledge, Restaurant Management Knowledge, Virtual Reality Experience, and Video Game Playing Experience. The Likert-based survey ranged from 0 to 4 in which the students represented their knowledge in each topic as either none (0), basic (1), average (2), above average (3), or expert (4) (see Figure 7). 

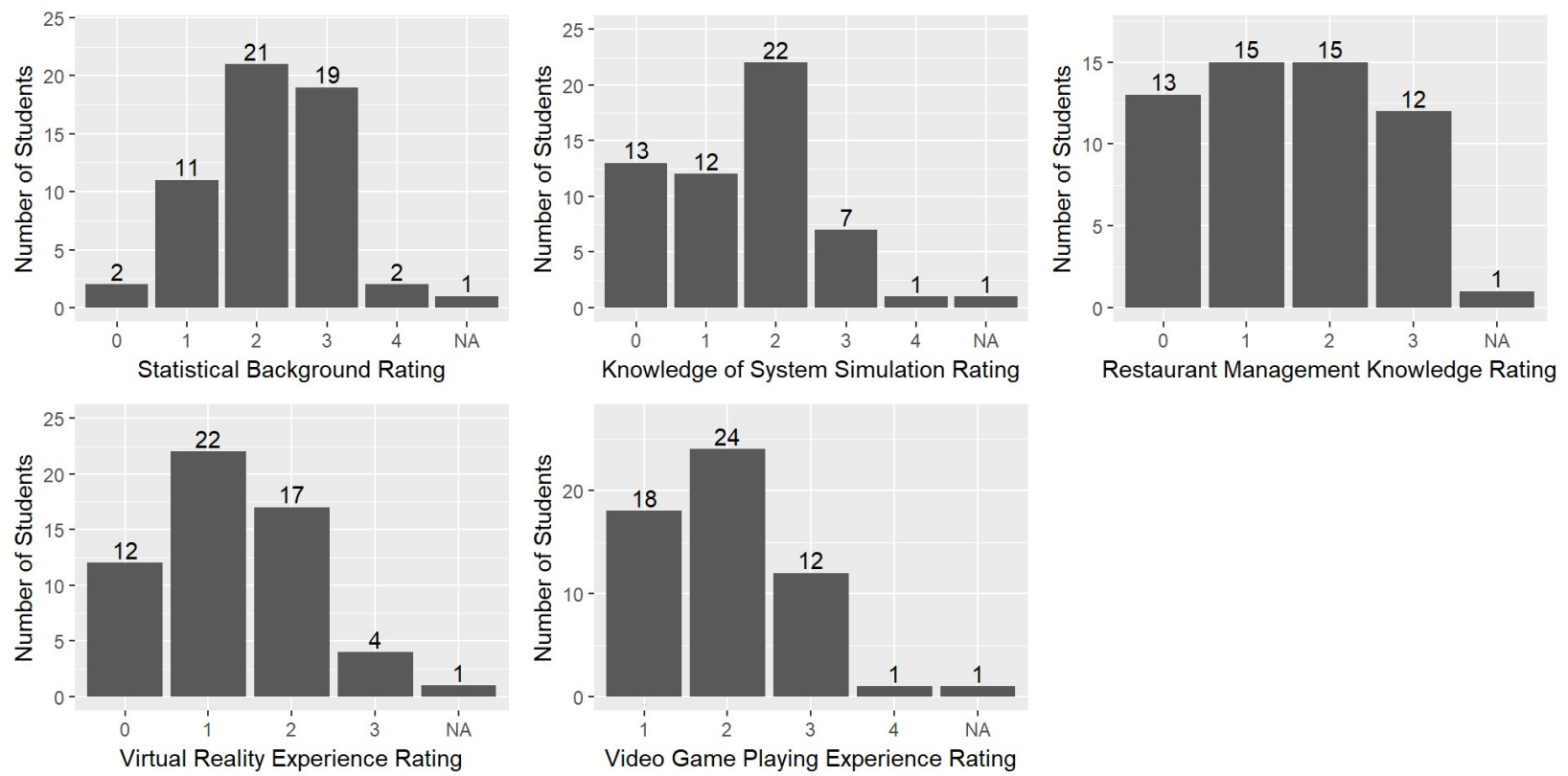

Figure 7: Distribution of Knowledge of Students

$60 \%$ of the students rated their statistical knowledge skills as average, while the other $40 \%$ perceived their statistical skills as above average. $40 \%$ of the students had an average understanding of system simulation, but $44 \%$ of the students had little to no knowledge of system simulation. In regards to student's understanding of restaurant management, the knowledge was somewhat uniformly distributed across the spectrum, with the caveat that no one considered themselves as an expert. For virtual reality and video game playing expertise, $60 \%$ and $75 \%$ respectively had either basic or no experience with either topic area.

\section{Dependent Variables and NASA TLX Assessment}

The evaluated dependent variables were the Overall Quiz Score, Conceptual Quiz Score, Analytical Quiz Score, NASA-TLX overall score, and each of the NASA-TLX five aspects of performance. The quiz consisted of 20 questions that tested students' knowledge of single and multi-queuing theory. Fifteen questions were designed to test the student's conceptual knowledge and five questions were designed to test their analytical knowledge of a special case of batch queuing, which was discussed in the virtual teaching module. The scores were calculated based on correctly answered percentage ratios for each quiz score type (See Appendix A). The perceived workload of the quiz was evaluated using the NASA Task Load Index (NASA-TLX). NASA-TLX is a subject tool that measures perceived workload when performing based on 6 aspects of performance [8], [13]:

- Mental Demand - measures the mental effort required to perform the task

- Physical Demand - measures the physical effort required to perform the task 
- Temporal Demand - measures the perceived time pressure to perform the task

- Overall Performance - measures the level of accomplishment in completing the overall task

- Effort - measures overall the level of effort, both physically and mentally, to perform the task

The NASA TLX assessment is divided into two phases. The first phase is where the subjects select on a rating scale from $1-100$ of how each aspect of design contributed to the overall impact of their perceived work effort to perform the quiz. The second phase is where the subjects are shown two aspects of performance at a time and they are required to select the aspect that was more of a contributor to their perceived work effort. After this is competed for each aspect of performance pairing, the aspect of performance contribution is weighted and the overall score is provided with an index score between $0-100$. The lower the index score correlates with lower perceived work load for the given task. Each aspect is rated on a $(0-100)$ scale and is combined to provide an overall task load index.

The NASA TLX quiz program used in this study can be reviewed at the website link: https://www.keithv.com/software/nasatlx/nasatlx.html. R (version 3.3.1) was used to conduct the data analysis for the study with the integrated development environment RStudios. R is a statistical computer environment and language that was developed by Bell Laboratories. It provides a large variety of statistical and graphical capabilities and is an open sources product [14].

\section{Results and Data Analysis}

The quiz scores are shown in Table 1 and Figure 8

Table 1: Quiz Score Statistics

\begin{tabular}{|c|c|c|c|c|c|c|}
\hline \multicolumn{6}{|c|}{ Quiz Scores Statistics } & \multirow[b]{2}{*}{ Ma: } \\
\hline & Min. & $\mathbf{1}^{\text {st }} \mathbf{Q u}$. & Median & Mean & $3^{\text {rd }} \mathbf{Q u}$ & \\
\hline Analytical & .20 & .40 & .60 & .6043 & .80 & 1 \\
\hline Conceptual & .27 & .60 & .80 & .7628 & .93 & 1 \\
\hline Overall & .25 & .575 & .70 & .7085 & .85 & 1 \\
\hline
\end{tabular}

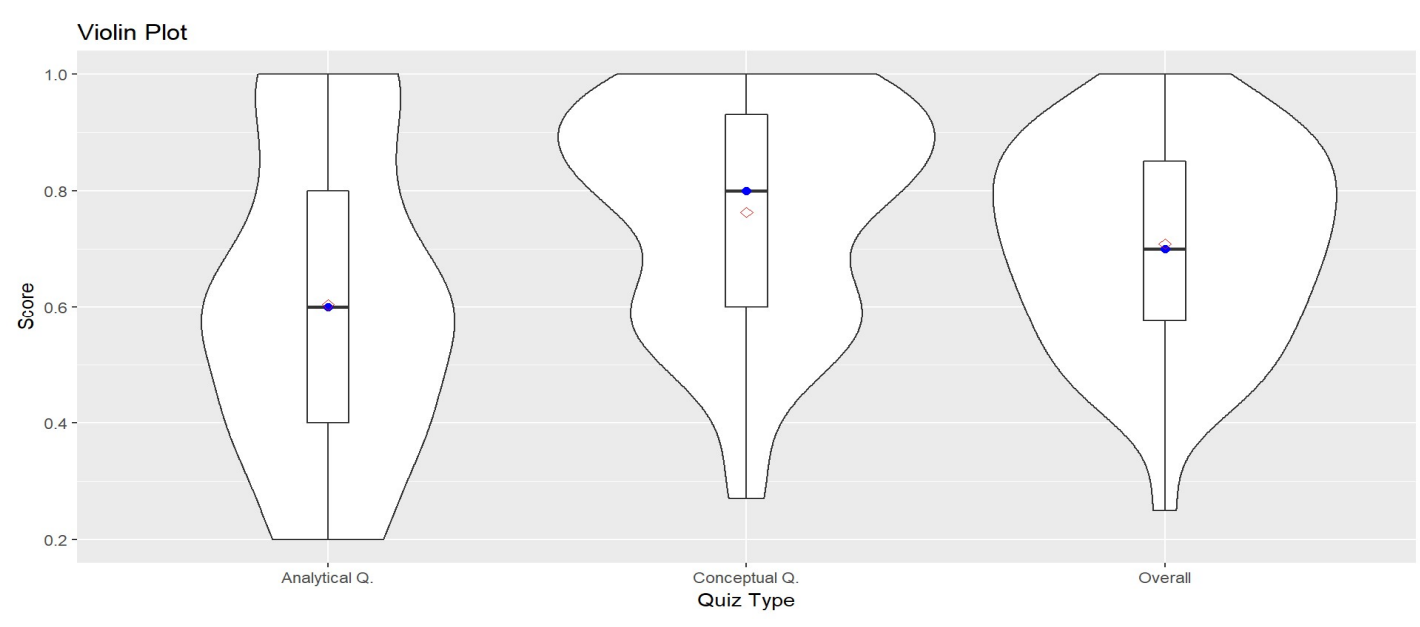


Figure 8: Quiz Score Statistics Violin Plot

For the overall score, the median and mean of scores were approximately $70 \% .50 \%$ of the scores ranged from $57.5 \%$ to $85 \%$. The students' average score was the highest for the conceptual proportion of the quiz. $50 \%$ of the scores ranged from $60 \%$ to $93 \%$ in which the center point of the data score was $80 \%$. Students performed worse on the analytical portion of the quiz, in which the average score was $60.43 \%$ and $50 \%$ of the data ranged from $40 \%$ to $80 \%$.

The shape of the violin plot shows the density of the scores within a score range. The analytical score plot is top-skewed and the conceptual and overall score plots are bottom-skewed. For the conceptual quiz, the plot is heavily bottom-skewed in which the scores tended to be clustered mostly on the higher end of above $80 \%$, even though the mean was $76.28 \%$. In this case, $25 \%$ (14 students) scored $93 \%$ or higher on the conceptual part of the quiz. For the analytical and overall plots, the plots appear slightly skewed in each respective direction. Figure 9 shows the correct answer ratio for each of the 20 quiz questions.

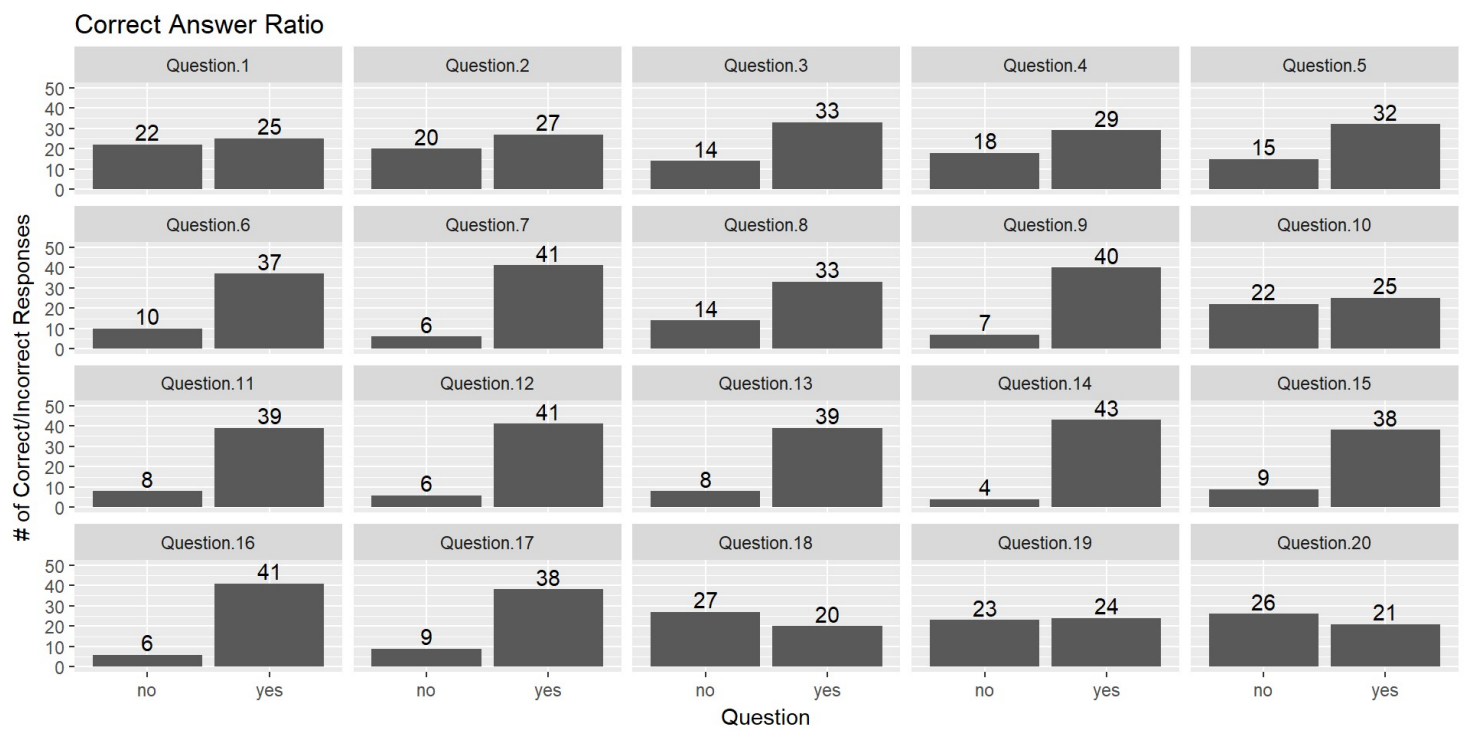

Figure 9: Correct Answer Ratio for Quiz Questions

The results show how well the students performed on each of the quiz questions. Most students did well on questions 3, 6-9, and 11-17, but for the other questions the results were approximately a 50\% chance of getting the answer correct. Specifically, for questions 18 and 20, more students answered incorrectly. Forty-seven students completed NASA TLX portion of the study. This is 
due to nine students having to stop the simulation due to simulation sickness. Table 2 and Figure 10 below, shows the results of the overall NASA TLX results

Table 2: NASA TLX Results

\begin{tabular}{|c|c|c|c|c|c|c|}
\hline \multicolumn{6}{|c|}{ Quiz Scores Statistics } & \multirow[b]{2}{*}{ Max } \\
\hline & Min. & $1^{\text {st }} \mathbf{Q u}$. & Median & Mean & $3^{\text {rd }} \mathbf{Q u}$ & \\
\hline $\begin{array}{c}\text { NASA TLX Index } \\
\text { Score }\end{array}$ & 11 & 39.50 & 56.50 & 55.57 & 74 & 97 \\
\hline
\end{tabular}

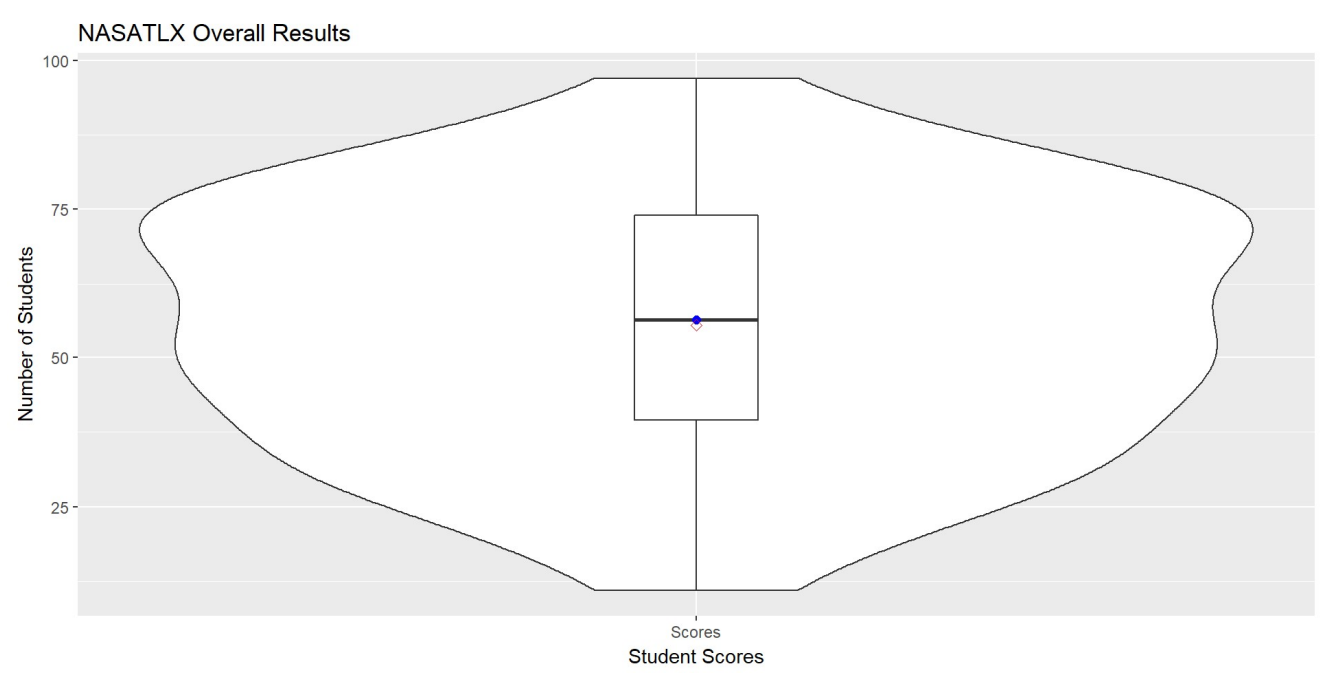

Figure 10: NASA TLX Overall Results Violin Plot

Overall, the average and median index score was appropriately 55\%. This concludes that the quiz required on average $55 \%$ perceived work effort to answer the quiz questions after completing the teaching module. Fifty percent of the scores ranged from $39.50 \%$ to $74 \%$ of perceived work load. The violin plot and box plots were not skewed and the scores seem normally distributed across the score spectrum. Figure 11 displays the results from the aspects of performance. 


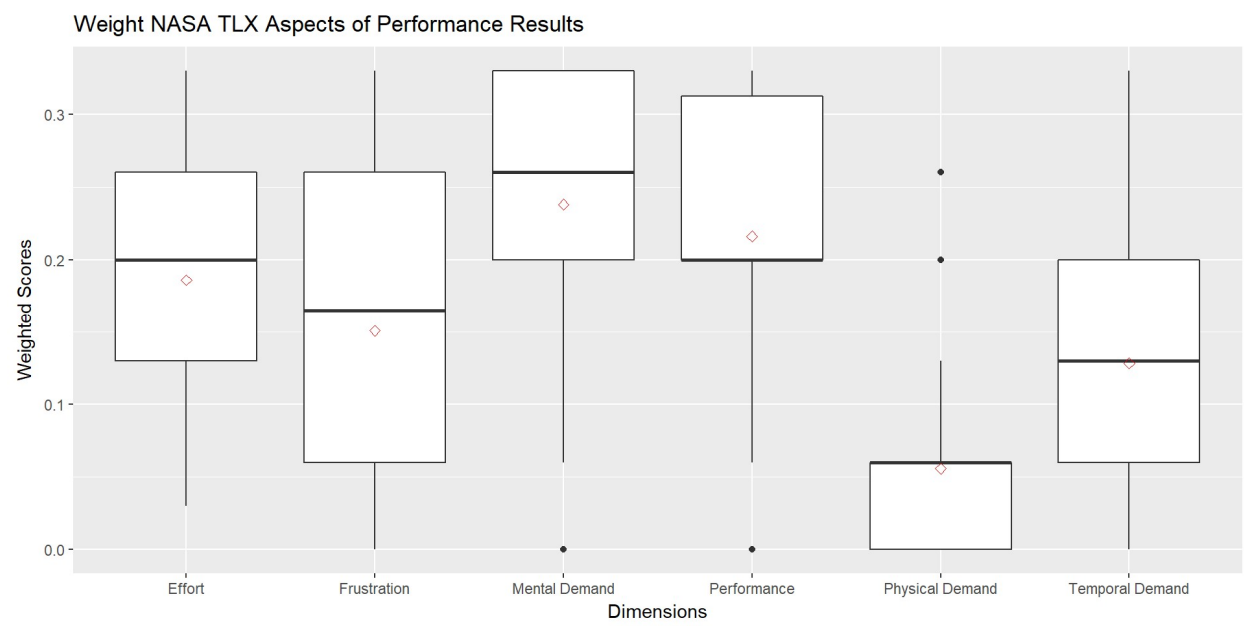

Figure 11: NASA TLX Aspects of Performance

The results indicate that, on average, mental demand and performance represented the largest contributors of the overall index scores. Physical demand was the lowest contributor of the overall perceived work effort followed by temporal demand as the second lowest. Both effort and frustration represented at least $15 \%$ of the contribution of perceived work effort in regards to perform the quiz task.

The Shapiro-Wilk normality test was conducted on the index scores with the result $\mathrm{W}=.97452$ and $p$-value 0.4623 . Since the $p$ value was not $<.05$, we could imply that the distribution of the data was not significantly different from the normal distribution. A one-way Analysis of Variance (ANOVA) was used to evaluate the overall index scores against the independent variables. The results are presented in Tables 3 and 4 . Two or more factor interactions were omitted for this effort.

Table 3: Overall Index Score ANOVA Results

\begin{tabular}{|c|c|c|c|}
\hline Source & DF & F & P \\
\hline Statistical Background & 4 & 1.39 & .255 \\
\hline Sys. Simulation Knowledge & 3 & .963 & .421 \\
\hline Restaurant Man. Knowledge & 3 & 1.72 & .179 \\
\hline Virtual Reality Exp. & 3 & 1.98 & .133 \\
\hline Video Game Exp. & 3 & .239 & .869 \\
\hline Academic Class. & 5 & 2.86 & .03 \\
\hline College Major & 11 & 1.77 & .105 \\
\hline Gender & 2 & 1.019 & .37 \\
\hline Race & 4 & 1.78 & .154 \\
\hline Origin & 1 & .047 & .83 \\
\hline
\end{tabular}


Table 4: Academic Classification Tukey Results

\begin{tabular}{|c|c|c|c|c|}
\hline Classification & Diff & Lwr & Upr & $\begin{array}{c}\text { P } \\
\text { Adj }\end{array}$ \\
\hline Undergraduate - Graduate & -15.58 & -30.24 & -.91 & 0.03 \\
\hline
\end{tabular}

The results indicate that there was a significant difference between the academic classification and the overall index score. The difference in the overall index score was significant between undergraduate and graduate students in which there was a 19.23-point difference in index scores. Senior students averaged 19 points lower than graduate students on the overall NASA TLX score. One-way factor ANOVAs for the aspects of performance weighted contribution was also evaluated. Tables 5 and 6 show the significant ANOVA and Tukey results for the relevant sources.

Table 5: Aspects of Performance Significant ANOVA Results

\begin{tabular}{|c|c|c|c|}
\hline \multicolumn{5}{|c|}{ Temporal Demand } \\
\hline Source & DF & F & P \\
\hline System Sim. Knowledge & 3 & 4.72 & $<.05$ \\
\hline VR Experience & 3 & 4.65 & $<.05$ \\
\hline \multicolumn{4}{|c|}{ Effort } \\
\hline VR Experience & 3 & 2.86 & $<.05$ \\
\hline \multicolumn{5}{|c|}{ Mental Demand } \\
\hline Academic Classification & 1 & 5.24 & $<.05$ \\
\hline
\end{tabular}

Table 6: Aspects of Performance Tukey Results

\begin{tabular}{|c|c|c|c|c|}
\hline \multicolumn{5}{|c|}{ Temporal Demand - System Simulation Knowledge Significant Tukey Results } \\
\hline Classification & Diff & Lwr & Upr & P-Adj \\
\hline $3-1$ & .15 & .04 & .27 & .003 \\
\hline $3-2$ & .11 & .01 & .22 & .022 \\
\hline \multicolumn{5}{|c|}{ Temporal Demand - Virtual Reality Knowledge Significant Tukey Results } \\
\hline Classification & Diff & Lwr & Upr & P-Adj \\
\hline $2-0$ & .09 & .01 & .18 & .02 \\
\hline $2-1$ & .09 & .02 & .17 & .008 \\
\hline \multicolumn{5}{|c|}{ Effort - Virtual Reality Knowledge Significant Tukey Results } \\
\hline Classification & Diff & Lwr & Upr & P-Adj \\
\hline $3-2$ & .11 & -.013 & .24 & .09 \\
\hline \multicolumn{5}{|c|}{ Mental Demand - Academic Classification Tukey Results } \\
\hline Classification & Diff & Lwr & Upr & P-Adj \\
\hline Undergraduate - & .07 & .009 & .145 & .027 \\
Graduate & \multicolumn{4}{c}{} \\
\hline
\end{tabular}


A significant difference was found between system simulation and virtual reality knowledge levels and the temporal demand weight scores. Virtual reality weighted scores were also found to be significantly different based on virtual reality knowledge. The Tukey result shows that above average knowledge in system simulations group temporal demand weighted scores were, on average, $15 \%$ higher than basic knowledge system simulation group. Also, the temporal demand weighted scores were, on average, $11 \%$ higher than the basic knowledge system simulation group. The results also show that the above average virtual reality knowledge groups weight temporal demands scores were $9 \%$ higher than both basic and no virtual reality knowledge groups. Even though virtual reality experience was found to be significant as a main effect, the Tukey results reveal that the factor was not truly significant between the virtual reality knowledge groups when $\mathrm{p}$ was adjusted for multiple comparisons. Lastly, the ANOVA results reveal a significant difference in mental demand weight scoring between academic classification groups. Undergraduate's mental demand weight score was $7 \%$ higher than graduate students in regards to the overall work load contribution.

\section{Discussion and Conclusion}

In regards to investigating conceptual knowledge after conducting a virtual reality teaching module, the scores on the quiz conclude that learning queuing theory virtual modules are feasible. The overall scores were lower than expected, given that the students could re-watch tutorial sections during the quiz portion of the tutorial. The conceptual knowledge quiz scores met the expectations, but it seemed that the students struggled with the analytical portion of the quiz. This is shown in the correct answer ratio graph (Figure 9) in which questions 10 and 17-20 represented the analytical question results. We concluded that part of the VR teaching design aspect negatively impacted the analytical results. Students provided some complaints about the use of the calculator in the analytical questions portion.

During the data collection process, we noticed two design factors that could have impacted the quiz results. The first factor was during the batch queuing portion of the tutorial. The equations were discussed and shown to the students in a step by step process. Based on the equation, it was designed to animate and move to the area of the simulation to the object that the equation represented analytical information of interest. For instance, the utilization equation moved from the center of the scene to the servers to represent the servers' utilization. Lambda moved from the center of the scene to the entry door of the customers, because it represents inter-arrival rate. The goal was to provide additional context to the equations by placing them near the objects. In hindsight, it seemed to have confused the students and also added to the possible simulation sickness to the participants.

The second factor pertained to the way the re-watch module was designed, and to the absence of in-simulation whiteboard capability for notes. During the quiz potion, students were able to rewatch sections of the simulation to review their knowledge. For the analytical questions, it would 
replay the flying portion of the scene again, and students would have to move from the quiz section to the area to re-watch the scene. In the next version of the VR module, we will design the equations to appear next to subjects (unanimated) as a reference to the subjects. Also, a virtual whiteboard will be provided to help students take notes and write results.

In the current design (phase II), subjects were required to come out of the VR environment and use paper to solve some of the questions. We concluded that exiting and reentering the environment impacted the subject's frustration levels as well as added to the simulation sickness factor as well. We are in the process of making changes to the design factors and further studies will be performed to investigate improvements in the conceptual knowledge of the students (Phase III).

The overall NASA TLX results showed that the students perceived the conceptual quiz to require average workload. The results from the ANOVA for the performance aspects were unexpected in that students with more knowledge in system simulation and virtual reality experience weighted temporal demand and effort higher than students with less knowledge in those areas. We concluded that students with more knowledge tried harder to perform well on the quiz and felt pressured because of how long it took to complete the quiz. Students with lesser knowledge in the area did not seem to allow the stress of the effort or time pressuring on the exam to affect them when conducting the quiz. In regards to the mental demand, undergraduate weighted mental demand as more on a contribution to the workload of the quiz. This is expected due to the lack of experience in the student's domain, undergraduates spent more time processing the quiz than the graduate students.

The difficulty of mapping students' TLX scores to their conceptual quiz scores is considered a limitation in the study. Thus, we were unable to compare the NASA TLX scores against how well the student performed on the quiz. The design feature is being added to the simulation and further studies will be conducted to investigate this research interest. Using immersive virtual teaching seems to be a viable option for teaching or enhancing students' knowledge in industrial engineering domain. Further studies are underway to investigate how well students perform using virtual teaching module versus traditional teaching methods. This will provide insight in understanding if the virtual training modules should be used as enhancement of students' traditional learned topics or could possibly be used as a standalone method of learning. Both would be beneficial to students, because it provides multiple avenues of learning to accommodate students who learn both conceptually and visually [15].

In terms of the VR system efficacy, three measurements were performed, 1) simulation sickness through three symptoms nausea, oculomotor disturbance, and disorientation, 2) VR Systems Usability - through comfort and ease of use, and 3) User Experience - through involvement, immersion, visual fidelity, interface quality, and sound. Simulation sickness analysis showed that the current VR teaching modules need some adjustments. The analysis of the systems usability 
and user experience of the module were found to be acceptable. In phase III of the research, we will improve the VR module to make a full self-paced tutorial where the instructor's role will be more facilitator than an instructor. 


\section{References}

[1] B. Dalgarno, A. G. Bishop, W. Adlong, \& D. R. Bedgood, (2009). "Effectiveness of a virtual laboratory as a preparatory resource for distance education chemistry students." Computers \& Education, 53(3), 853-865.

[2] C. Dede, M. C. Salzman, R. B Loftin, \& D. Sprague. (1999). "Multisensory immersion as a modeling environment for learning complex scientific concepts” In W. Feurzeig, \& N. Roberts (Eds.), Computer modeling and simulation in science education (pp. 282-319). New York: Springer-Verlag.

[3] M. Bricken, \& C. M. Byrne. (1994). "Summer students in virtual reality: A pilot study on educational applications of virtual reality technology." In A. Wexelblat (Ed.), Virtual reality: Applications and explorations (pp.199-218), Boston, MA: Academic.

[4] J. K. Crosier, S. V. G. Cobb, J.R. Wilson. (2000). "Experimental comparison of virtual reality with traditional teaching methods for teaching radioactivity". Education and Information Technologies, 5 (4), pp. 329-343.

[5] T. A. Mikropoulos, and J. Bellou, (2006). "The unique features of educational virtual environments.” Proceedings E-Society, pp. 122-128.

[6] P. C. Smith, and B. K. Hamilton. (2015). "The Effects of Virtual Reality Simulation as a Teaching Strategy for Skills Preparation in Nursing Students." Clinical Simulation in Nursing, 11(1), pp.52-58.

[7] A. William, V. L. Vidal, \& P. John. (2016). “Traditional Instruction versus Virtual Reality Simulation: A Comparative Study of Phlebotomy Training among Nursing Students in Kuwait." Journal of Education and Practice, 7(9), 18-25.

[8] L. Colligan, H.W.W. Potts, C.T. Finn, and R. A. Sinkin. (July 2015). "Cognitive workload changes for nurses transitioning from a legacy system with paper documentation to a commercial electronic health record." International Journal of Medical Informatics. 84 (7): 469-476.

[9] B. Dalgarno, J. Hedberg, and B. Harper. (2002). "The contribution of 3D environments to conceptual understanding." In Proceedings of the 19th Annual Conference of the Australian Society for Computers in Tertiary Education (ASCILITE). Auckland, New Zealand: UNITEC Institute of Technology, Auckland, New Zealand. Retrieved July 16, 2009, from http://www.ascilite. org.au/conferences/ auckland02/proceedings/papers/051.pdf

[10] Baillie, Goodhew, and Skryanbina (2006) "Threshold Concepts in Engineering Education Exploring Potential Blocks in Student Understanding." https://www.ijee.ie/articles/Vol22-5/06 ijee1823.pdf

[11] Ausubel. “Ausubel's Assimilation Learning Theory: Theoretical Basis for Concept Maps and E-Maps" http://etec.ctlt.ubc.ca/510wiki/Ausubel's_Assimilation_Learning_Theory:_Theoretical_B asis_for_Concept_Maps_and_E-Maps 
[12] D. G. Kendall. (1953). "Stochastic Processes Occurring in the Theory of Queues and their Analysis by the Method of the Imbedded Markov Chain". The Annals of Mathematical Statistics. 24 (3): 338. JSTOR 2236285. doi:10.1214/aoms/1177728975.

[13] NASA (1986). Nasa Task Load Index (TLX) v. 1.0 Manual

[14] https://www.r-project.org/about.html

[15] M. Roussou. (2004). Learning by Doing and Learning Through Play: An Exploration of Interactivity in Virtual Environments for Children. Comput. Entertain., 2(1), pp. 1-10. 


\section{Appendix A}

\section{Conceptual Knowledge Questionnaire}

1. What is concept of Queuing Theory?
a. Forces applied to an object
b. Waiting lines
c. Rate of Service Speed
d. Entity Arrival Rate

2. What symbol denotes average wait time in system in queue
a. $\rho$
b. $\lambda$
c. $\mathrm{W}$
d. $\mathrm{L}_{\mathrm{q}}$
e. $\mathrm{W}_{\mathrm{q}}$

3. What symbol denotes average server utilization
a. $\rho$
b. $\lambda$
c. $\mathrm{W}$
d. $\mathrm{L}_{\mathrm{q}}$
e. $\mathrm{W}_{\mathrm{q}}$

4. What symbol denotes average number of customers in queue
a. $\rho$
b. $\lambda$
c. $\mathrm{W}$
d. $\mathrm{L}_{\mathrm{q}}$
e. $\mathrm{W}_{\mathrm{q}}$

5. What symbol denotes the arrival rate of customers in the system
a. $\rho$
b. $\lambda$
c. $\mathrm{W}$
d. $\mathrm{L}_{\mathrm{q}}$
e. $\mathrm{W}_{\mathrm{q}}$

6. What symbol denotes average wait time in system
a. $\rho$
b. $\lambda$
c. $\mathrm{W}$
d. $\mathrm{L}_{\mathrm{q}}$
e. $\mathrm{W}_{\mathrm{q}}$

7. Assuming there are two servers in the system. Server 1 utilization is .66 and Server 2 utilization is .80. Which servers is utilization the most?
a. Server 1
b. Server 2 
8. Based on the question above, if another server was added to the staff, pick the correct effect below:

a. Server 1 Utilization would go up, Server 2 Utilization would go Down

b. Server 1 Utilization would go Down, Server 2 Utilization would go Up

c. Server 1 Utilization would go up, Server 2 Utilization would go Up

d. Server 1 Utilization would go Down, Server 2 Utilization would go Down

9. In a grocery store environment and you are the customer, who would represent the a server
a. Cashier
b. Other customers
c. Buggy
d. Store Door

10. Which customer arrival rate would be the fastest
a. 2 customers / $\mathrm{min}$
b. 80 customers / hour
c. 1 customers / $40 \mathrm{secs}$
d. 2000 customers / day

11 . What does being $50 \%$ utilized mean?

a. The server is working $50 \%$ above maximum workload

b. The server is working $50 \%$ of the time

c. The server is idle

d. The server is a maximum workload

12. If the arrival rate was 2 customers / min and the server rate 1 customer / min, will steady state (a stabilization of the queue, it not continuing to grow) be reach. ?
a. Yes
b. No

13. If the arrival rate was 5 customers / min and server rate 3 customer / min, will steady state be reached?
a. Yes
b. No

14. What is the utilization of a server that doesn't get a break?
a. $50 \%$
b. $0 \%$
c. $100 \%$
d. $70 \%$

15. What is the utilization of a server that is always idle?
a. $50 \%$
b. $0 \%$
c. $100 \%$
d. $70 \%$ 
16. What is the utilization of 4 servers equally utilized
a. $25 \%$
b. $50 \%$
c. $75 \%$
d. $100 \%$

\section{Equations}

Average Service Utilization: $\rho=\frac{\lambda}{s \mu}$

Average \# of customers: $L_{q=\frac{\rho^{2}}{(1-\rho)}}$

Average waiting time in queue: $W_{q}=\frac{L_{q}}{\lambda}$

Average time customer spends in the system: $W=W_{q}+\frac{1}{\mu}$

\section{Question}

The arrival rate of the McDonalds is 7 customers per minute and the service rate is 3 customers per minute. The current system has 3 servers to service the customers. Answer the following questions

17. What is the average service utilization?
a. $40 \%$
b. $78 \%$
c. $95 \%$
d. $1.05 \%$

18. What is the average number of customers in the queue
e. 2.7
f. 5.2
g. 3.9
h. 1.2

19. What is the average waiting time in the queue

i. 2.3

j. .33

k. .39

1. 0

20. What is the average time customer spend in the system
m. .76
n. .85
o. 1
p. .72 